\title{
On the robust controller design for Hard Disk Drive servo systems with time delays
}

\author{
Peng Yan ${ }^{1}$, Hitay Özbay ${ }^{2}$
}

\begin{abstract}
Due to the existence of various sources of delays, the dynamical model of HDD (Hard Disk Drive) servo systems is actually infinite dimensional, although most of the control algorithms simplified the model with Padé expansions or other finite dimensional approximations. In this paper, a robust loop shaping algorithm is developed for the HDD model with delays by using an $\mathcal{H}^{\infty}$ synthesis approach for infinite dimensional systems. The $\mathcal{H}^{\infty}$ controller is derived with a structure of an internal feedback loop including an FIR (Finite Impulse Response) filter and an IIR (Infinite Impulse Response) filter, which facilitates non-fragile implementations. Comparisons to other robust control methods are given and the advantages of this approach are demonstrated in terms of improvement of TMR (track misregistration) and tracking TPI (Track-perInch) capability.
\end{abstract}

\section{INTRODUCTION}

The rapid growth of research on nano-precision mechatronics and the immense demands of HDD storage with various application have imposed many new challenges on servo technologies. There have been considerable research works that address important control issues inherited in this area, see [1], [3], and [4]. In [22] a robust $\mu$-synthesis approach is introduced for the head positioning of a VCM (Voice Coil Motor) actuator in HDD. The robust loop shape problem is also discussed in [8], where a dual stage actuation architecture is adopted. Track-seeking is also an important topic inviting considerable studies, for example, the wellknown PTOS (Proximate Time Optimal Seek) control approach discussed in [16], [17], and more recent result on the use of variable structure observer in seeking control [15]. Advanced control algorithms are not only used in servo operations for data access, but also in the process of writing servo patterns during HDD manufacturing, see for example [20], [5], [14].

Among the abundant research results on HDD servo algorithms, one of the central topics is the robust loop shaping for HDD servo systems, which determines the servo bandwidth, attenuations of disturbance, and transient behaviors during seek settling. Robust control theory has been widely investigated for HDD [22], [8], as well as other nano-positioning applications [9], [18], [10], [25], where standard $\mathcal{H}^{\infty}$ control algorithms and $\mu$-synthesis [24] have been successfully developed. It is worth noting that the dynamical model of

\footnotetext{
1 School of Automation Science and Electrical Engineering, Beihang University, Beijing, 100191, China; also with Key Laboratory of Highefficiency and Clean Mechanical Manufacturing, Ministry of Education, School of Mechanical Engineering, Shandong University, Jinan, Shandong, 250061, P. R. China PengYan2007@gmail. com

2 Bilkent University, Electrical and Electronics Engineering Department, 06800, Bilkent, Ankara, Turkey hitay@bilkent.edu.tr
}

HDD servo systems is actually infinite dimensional due to the existence of various sources of delays [21], although most of the control algorithms simplified the model with Padé expansion or other finite dimensional approximations. Therefore two important questions remain open in this area: 1 ) is it possible to synthesize robust $\mathcal{H}^{\infty}$ controllers for the infinite dimensional HDD model directly, and 2) what are the advantages of the direct approach, compared to the existing approximation methods?

In this paper, the time delay model of HDD servo systems is analyzed with uncertainties and formulated to a mixed sensitivity optimization problem for robust control. By using the $\mathcal{H}^{\infty}$ control theory for infinite dimensional systems [6], the optimal $\mathcal{H}^{\infty}$ performance can be numerically computed and an explicit form of the optimal $\mathcal{H}^{\infty}$ controller can be developed. Based on coprime-inner/outer factorization, we can eliminate the unstable pole-zero cancelations in the $\mathcal{H}^{\infty}$ controller and generate the controller structure with an internal feedback loop including an FIR (Finite Impulse Response) filter and an IIR (Infinite Impulse Response) filter, [7], [12], [23], which facilitates a non-fragile implementation of the robust servo controller. Another important contribution of the present paper is the comparisons to existing robust control methods, which demonstrate significant improvement of TMR and tracking TPI (Track-per-Inch) capability.

The rest of the paper is organized as follows: in Section II, we describe the HDD servo model with time delays and uncertainties. In Section III, we discuss the mixed sensitivity optimization problem for the SISO time delay systems, and the non-fragile structure for controller implementations. A design case is studied in details in IV, where comparisons are also provided with existing robust control approaches of Padé approximations. Tracking TPI capabilities and TMR analysis are discussed in Section $\mathrm{V}$ to illustrate the advantages of the proposed design, followed by conclusions in Section VI.

\section{MODELING OF HDD SERVO SYSTEMS}

A modern HDD can be considered as an ultra-high precision mechatronics device. The dynamics of a single stage HDD with a VCM actuator can be modeled as:

$$
P(s)=\frac{K_{D C} e^{-h s}}{s^{2}} T_{s}(s) T_{h}(s),
$$

where $K_{D C}$ is the actuator DC gain, $h$ the total time delay from various sources such as PWM filters, power amplifiers, actuator delays, and $T_{s}(s)$ the first translational 

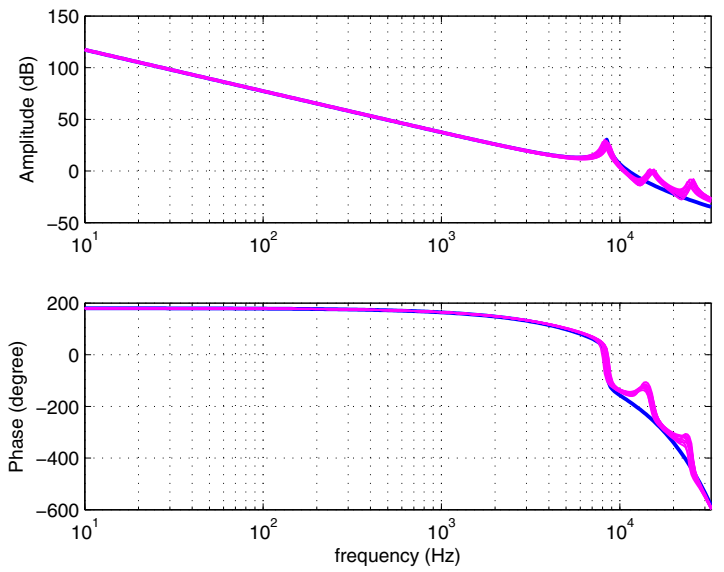

Fig. 1. Nominal plant and actual plants with uncertainties.

mode (system mode):

$$
T_{s}(s)=\frac{A_{s}(s)}{B_{s}(s)}=\frac{s^{2}+2 \xi_{z, 0} \omega_{n_{z, 0}} s+\omega_{n_{z, 0}}^{2}}{s^{2}+2 \xi_{p, 0} \omega_{n_{p, 0}} s+\omega_{n_{p, 0}}^{2}},
$$

where $\xi_{z, 0}$ and $\omega_{n_{z, 0}}$ are the damping ratio and natural frequency of the zeros of the system mode, and $\xi_{p, 0}$ and $\omega_{n_{p, 0}}$ the damping ratio and natural frequency of the poles. Note that $T_{s}(s)$ is stable, biproper and minimum phase, i.e., $T_{s}(s) \in \mathcal{H}^{\infty}$ and $T_{s}^{-1}(s) \in \mathcal{H}^{\infty}$, which is usually guaranteed by the mechanical design of HSA (Head Suspension Assembly). All the high frequency resonant modes can be modeled by $T_{h}(s)$ :

$$
T_{m}(s)=\prod_{i=1}^{N} \frac{\frac{1}{\omega_{n_{z, i}}^{2}} s^{2}+2 \frac{\xi_{z, i}}{\omega_{n_{z, i}}} s+1}{\frac{1}{\omega_{n_{p, i}}^{2}} s^{2}+2 \frac{\xi_{p, i}}{\omega_{n_{p, i}}} s+1},
$$

where $\xi_{z, i}, \xi_{p, i}, \omega_{n_{z, i}}$ and $\omega_{n_{p, i}}$ are the damping ratios and natural frequencies of the $i^{\text {th }}$ resonant mode.

We define the nominal model of (1) as:

$$
P_{0}(s)=\frac{K_{0} e^{-h s}}{s^{2}} \bar{T}_{s}(s),
$$

where

$$
\bar{T}_{s}(s)=\frac{\bar{A}_{s}(s)}{\bar{B}_{s}(s)}=\frac{s^{2}+2 \bar{\xi}_{z, 0} \bar{\omega}_{n_{z, 0}} s+\bar{\omega}_{n_{z, 0}}^{2}}{s^{2}+2 \bar{\xi}_{p, 0} \bar{\omega}_{n_{p, 0}} s+\bar{\omega}_{n_{p, 0}}^{2}} .
$$

As depicted in Figure 1, the nominal plant $P_{0}(s)$ is illustrated by the thick blue line and the actual plant with various uncertainties can be illustrated by the thin magenta lines. Now that the multiplicative uncertainties can be written as:

$$
\Delta P_{m}(s)=\frac{P(s)-P_{0}(s)}{P_{0}(s)}=\frac{A_{s}(s) \bar{B}_{s}(s)}{\bar{A}_{s}(s) B_{s}(s)} T_{m}(s)-1 .
$$

We denote the multiplicative uncertainty bound $W_{2}(s)$ with $\left|\Delta P_{m}(s)\right|_{s=j \omega} \leq\left|W_{2}(s)\right|_{s=j \omega}$, which can be used to describe the the upper and lower bounds of the plant variations by $\left|P_{0}(j \omega)\right| \pm\left|P_{0}(j \omega) W_{2}(j \omega)\right|$ (see the the dash lines in Figure 2).

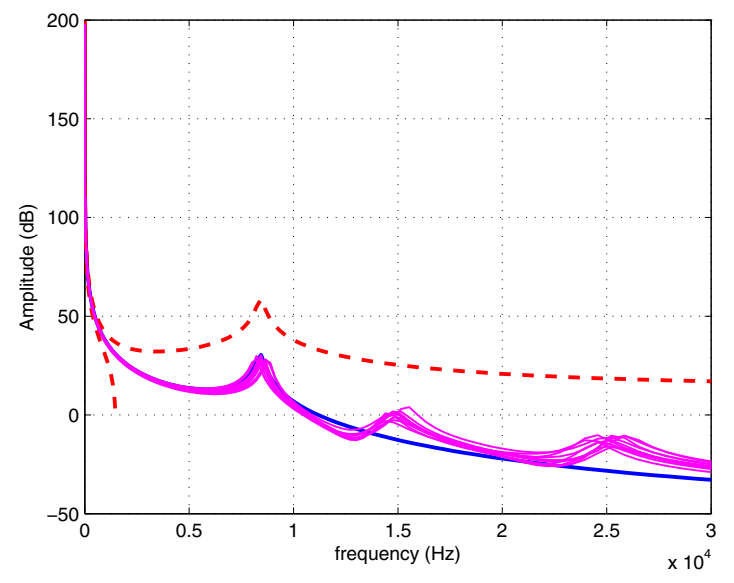

Fig. 2. Plant model and uncertainty bounds.

\section{MIXED SENSITIVITY OPTIMIZATION FOR INFINITE DIMENSIONAL SYSTEMS}

Various robust control algorithms has been widely discussed for HDD servo design (see [11], [13], [8], [21], to cite just a few contributions). An applicable $\mathcal{H}^{\infty}$ control method in servo loop shaping is mixed sensitivity optimization, where the nominal performance (determined by performance weighting $W_{1}(s)$ ) and robust stability (determined by uncertainty weighting $W_{2}(s)$ ) can be optimized simultaneously:

$$
\inf _{C s t a b . P_{0}}\left\|\left[\begin{array}{c}
W_{1}(s)\left(1+P_{0}(s) C(s)\right)^{-1} \\
W_{2}(s) P_{0}(s) C(s)\left(1+P_{0}(s) C(s)\right)^{-1}
\end{array}\right]\right\|_{\infty}
$$

Note that standard $\mathcal{H}^{\infty}$ control theory, as readily implemented in the robust control toolbox of Matlab, cannot be applied due to the existence of time delays in $P_{0}(s)$. In what follows, we would like to investigate the mixed sensitivity optimization problem (7) using the robust control theory for infinite dimensional systems developed in [6], [19] based on frequency domain techniques. For time delay systems considered here state-space based design methods are also available, [12], [23].

First, define

$$
\begin{aligned}
\tilde{P}_{0}(s) & =P_{0}(s) * \bar{T}_{s}^{-1}(s) \approx \frac{K_{0} e^{-h s}}{(s+\epsilon)^{2}} \\
\tilde{C}(s) & =C(s) * \bar{T}_{s}(s),
\end{aligned}
$$

where $\epsilon>0$ is sufficient small. With this, the original $\mathcal{H}^{\infty}$ optimization (7) can be written as:

$$
\inf _{\tilde{C} s t a b . \tilde{P}_{0}}\left\|\left[\begin{array}{c}
W_{1}(s)\left(1+\tilde{P}_{0}(s) \tilde{C}(s)\right)^{-1} \\
W_{2}(s) \tilde{P}_{0}(s) \tilde{C}(s)\left(1+\tilde{P}_{0}(s) \tilde{C}(s)\right)^{-1}
\end{array}\right]\right\|_{\infty},
$$

where the optimal $\mathcal{H}^{\infty}$ controller, denoted by $\tilde{C}_{\text {opt }}(s)$, yields:

$$
\left\|\left[\begin{array}{c}
W_{1}(s)\left(1+\tilde{P}_{0}(s) \tilde{C}_{o p t}(s)\right)^{-1} \\
W_{2}(s) \tilde{P}_{0}(s) \tilde{C}_{o p t}(s)\left(1+\tilde{P}_{0}(s) \tilde{C}_{o p t}(s)\right)^{-1}
\end{array}\right]\right\|_{\infty}=\gamma_{o} .
$$

We define $W_{1}(s)=K_{1} / s^{2}$ to meet the design specification of HDD servo loop on low frequency roll-off around 
$40 \mathrm{~dB} / \mathrm{dec}$ for disturbance attenuations. The multiplicative uncertainty weight is chosen as a second order polynomial $W_{2}(s)=a+b s+c s^{2}$ to accommodate model uncertainties (see Figure 2). Observe that $\tilde{P}_{0}(s)$ admits the coprime inner/outer factorization $\tilde{P}_{0}(s)=m_{n} N_{o}$, where $m_{n}=$ $e^{-h s} \in \mathcal{H}^{\infty}\left(\mathbb{C}_{+}\right)$is inner and $N_{o}=K_{0} /(s+\epsilon)^{2} \in \mathcal{H}^{\infty}\left(\mathbb{C}_{+}\right)$ is outer.

Applying the formulae of [6], [19], the optimal solution to (9) is given in the form:

$$
\tilde{C}_{\text {opt }}(s)=\frac{1}{K_{0}} E_{\gamma_{o}}(s) \frac{s^{2} F_{\gamma_{o}}(s) L(s)}{1+e^{-h s} F_{\gamma_{o}}(s) L(s)}
$$

where

$$
\begin{gathered}
E_{\gamma_{o}}(s)=\frac{n E(s)}{d E(s)}=\frac{K_{1}^{2}-\gamma_{o}^{2} s^{4}}{\gamma_{o}^{2} s^{4}} \\
F_{\gamma_{o}}(s)=\frac{n F(s)}{d F(s)}=\frac{s^{2}}{f_{4} s^{4}+f_{3} s^{3}+f_{2} s^{2}+f_{1} s+f_{0}}
\end{gathered}
$$

with coefficients $f_{0}, \ldots, f_{4}$ depending on $\gamma_{o}$

$$
L(s)=\frac{n L(s)}{d L(s)}=\frac{1+a_{L} s}{1-a_{L} s}
$$

with $a_{L}$ and $\gamma_{o}$ are determined from two interpolation conditions, see [19].

\section{A. Controller Structure and Implementation}

It is noticed that the $\mathcal{H}^{\infty}$ controller (10) has unstable zeropole cancelations due to interpolation conditions. However the exact cancelations for the factorization terms are not possible because of the infinite dimensional term of the time delay in the controller [7], [12], [23]. We would like to rearrange the terms in the controller to eliminate the unstable zero-pole cancelations such that the above controller structure is implementable.

In fact, $\tilde{C}_{\text {opt }}(s)$ can be rewritten as:

$$
\tilde{C}_{\text {opt }}(s)=\frac{1}{K_{0} f_{4}}\left(\frac{1}{1+H(s)}\right),
$$

where

$$
H(s)=H_{1}(s)+H_{F I R}(s),
$$

with

$$
H_{1}(s)=\frac{Q_{H_{1}}}{1+a_{L} s}, Q_{H_{1}}=\frac{2}{f_{4}} \frac{d F\left(-1 / a_{L}\right)}{n E\left(-1 / a_{L}\right)},
$$

It can be further shown that $H_{F I R}(s)$ is an FIR filter defined as:

$$
H_{F I R}(s)=\mathcal{L}\left\{h_{F I R}(t)\right\}
$$

with

$$
h_{F I R}(t)=\left\{\begin{array}{cl}
C_{0} e^{A_{0} t} B_{0} & 0 \\
0 & t>h
\end{array}\right.
$$

where

$$
C_{0}\left(s I-A_{0}\right)^{-1} B_{0}=\frac{h_{0}(s)}{\left(s+1 / a_{L}\right) n E(s)},
$$

and

$$
\begin{aligned}
h_{0}(s) & =\frac{1}{f_{4}}\left(1 / a_{L}-s\right) d F(s) \\
& -\left(1 / a_{L}+s\right) n E(s)-Q_{H_{1}} n E(s) .
\end{aligned}
$$

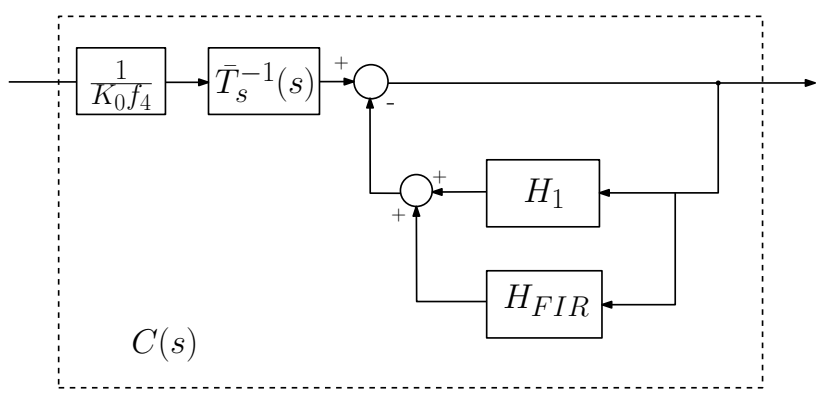

Fig. 3. Controller structure.

From (8), (12) and (13), we can derive the optimal $\mathcal{H}^{\infty}$ controller

$$
C_{\text {opt }}(s)=\frac{1}{K_{0} f_{4}} \bar{T}_{s}^{-1}(s)\left(\frac{1}{1+H_{1}(s)+H_{F I R}(s)}\right),
$$

which has the structure as depicted in Figure 3. It is worth noting that the controller structure (17) facilitates non-fragile digital implementations due to the FIR feature of $H_{F I R}(s)$ and the low order representation of $H_{1}(s)$.

\section{B. Padé Approximation Methods}

Most of the existing results on HDD servo loop shaping employ low order Padé approximations (18) to simplify the problem to a finite dimensional system, with which the closed form solutions of $\mathcal{H}^{\infty}$ controllers to the problem (7) can be synthesized by standard 2-block $\mathcal{H}^{\infty}$ optimization [24].

$$
e^{-h s} \approx T_{h}^{m}(s):=\frac{1-k_{1} s+k_{2} s^{2}+\cdots \pm k_{m} s^{m}}{1+k_{1} s+k_{2} s^{2}+\cdots+k_{m} s^{m}},
$$

where $T_{h}^{m}(s)$ is the $m^{t h}$ order Padé approximation.

Now that an important open problem is the conservativeness of this method using various Padé approximations and how they are compared with the direct method discussed above. For this purpose, we consider the mixed sensitivity optimization (7) by Padé approximations with $e^{-h s} \approx$ $T_{h}^{m}(s), m=1,2,3$, respectively. We denote

$$
P_{0}^{m}(s)=\frac{K_{0}}{s^{2}} T_{h}^{m}(s) \bar{T}_{s}(s), \quad m=1,2,3
$$

and

$$
\begin{aligned}
& \tilde{W}_{1}(s):=\frac{K_{1}}{\left(s+\epsilon_{1}\right)^{2}} \approx W_{1}(s), \quad \epsilon_{1}>0 \\
& \tilde{W}_{2}(s):=\frac{a+b s+c s^{2}}{\left(\epsilon_{2} s+1\right)^{2}} \approx W_{2}(s), \quad \epsilon_{2}>0 .
\end{aligned}
$$

A standard mixed sensitivity optimization problem can formulated for finite dimensional models $P_{0}^{m}(s), m=1,2,3$ :

$$
\inf _{C^{m} \text { stab. } P_{0}^{m}}\left\|\left[\begin{array}{c}
\tilde{W}_{1}\left(1+P_{0}^{m} C^{m}\right)^{-1} \\
\tilde{W}_{2} P_{0}^{m} C^{m}\left(1+P_{0}^{m} C^{m}\right)^{-1}
\end{array}\right]\right\|_{\infty}, m=1,2,3 .
$$

Note that low order Padé approximations result in inaccuracies at high frequency, while higher order approximations lead to high order controller dynamics and undesired zeroes/poles which could cause transient problem. We will use 


\begin{tabular}{|c|c|c|c|c|c|}
\hline$K_{0}$ & $h$ & $\xi_{z, 0}$ & $\xi_{p, 0}$ & $\bar{\omega}_{n_{z, 0}}$ & $\bar{\omega}_{n_{p, 0}}$ \\
\hline $5.2269 \times 10^{8}$ & $6 \times 10^{-5}$ & 0.99 & 0.018 & $1.244 \times 10^{5}$ & $5.29 \times 10^{4}$ \\
\hline \hline$\epsilon$ & $K_{1}$ & $a$ & $b$ & $c$ & \\
\hline 0.01 & $2 \times 10^{7}$ & 0.3125 & $9.4211 \times 10^{-6}$ & $8.772 \times 10^{-11}$ & \\
\hline
\end{tabular}

TABLE I

MODEL COEFFICIENTS.

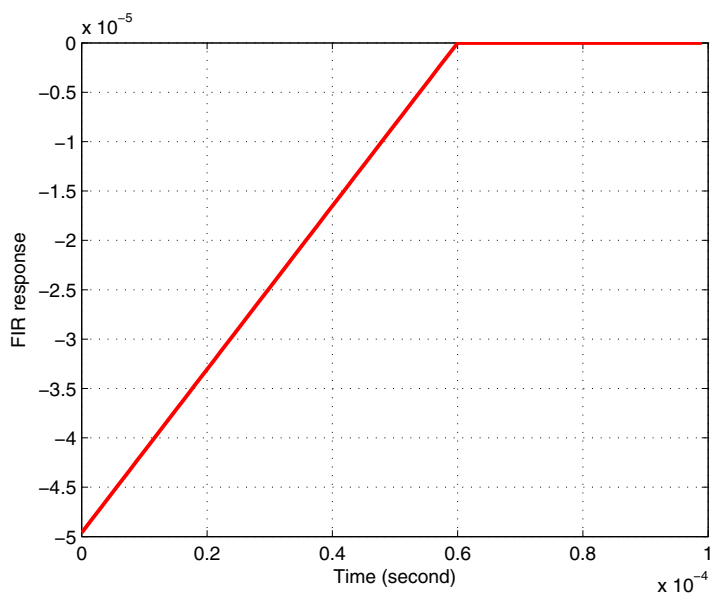

Fig. 4. Time response of FIR $H_{F I R}(s)$.

a design example to compare the direct method for infinite dimensional systems and the Padé approximation methods.

\section{A DESIGN CASE STUDY}

In this section, we apply the control algorithm derived in the above section to an industry design case, where an enterprize class 2.5 -inch form factor HDD is considered. The HDD has $15 k R P M$ rotation speed for high performance purpose, which requires challenging design specifications on the servo control system. The nominal plant model (4) and the weighting functions are defined in Table I. The nominal model (blue line) of the HDD VCM structures and the uncertainty bounds are depicted in Figure 2.

To begin with, we determine the optimal $\mathcal{H}^{\infty}$ performance $\gamma_{o}=0.48336$ using the computational procedure of [19]. That leads to the following numerical values for the components of the optimal $\mathcal{H}^{\infty}$ controller in Section III:

$$
\begin{gathered}
\bar{E}_{\gamma_{o}}(s)=\frac{n \bar{E}}{d \bar{E}}=\frac{-0.2336 s^{4}+4 \times 10^{14}}{0.2336 s^{4}}, \\
\bar{L}(s)=\frac{n \bar{L}}{d \bar{L}}=\frac{1+a_{L} s}{1-a_{L} s}, \quad a_{L}=2.5124 \times 10^{-4},
\end{gathered}
$$

and $f_{4}=1.81 \times 10^{-10}, f_{3}=2.127 \times 10^{-5}, f_{2}=0.8467$, $f_{1}=7294, f_{0}=3.156 \times 10^{7}$ in (11). From (13) to (17), we have the optimal $\mathcal{H}^{\infty}$ controller written as (21) where the FIR term $H_{F I R}(s)$ is determined by (15) and (16), with time domain response depicted in Figure 4.

For comparison purpose, we also synthesize $\mathcal{H}^{\infty}$ controllers based on (20) using various Padé approximations. As

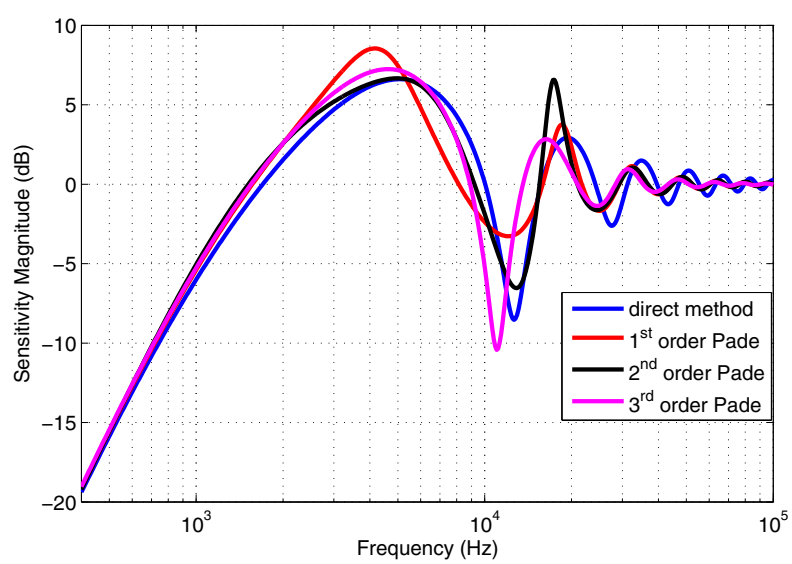

Fig. 5. Sensitivity comparisons.

depicted in Figure 5, the proposed direct method significantly improves the closed loop bandwidth compared to Padé approximation methods: $0 \mathrm{~dB}$ crossing occurs at $1701 \mathrm{~Hz}$ with the direct design, whereas the same occurs at $1565 \mathrm{~Hz}$, $1517 \mathrm{~Hz}$ and $1549 \mathrm{~Hz}$, for the 1 st, $2 n d$ and $3 r d$ order Padé approximation based designs, respectively. The direct design has the added advantage of lower sensitivity peaking, see Table II.

\begin{tabular}{||c||c|c||}
\hline Method & Sensitivity Peak & Peak Frequency \\
\hline \hline Direct & $6.61 \mathrm{~dB}$ & $5091 \mathrm{~Hz}$ \\
\hline Pade 1 & $8.55 \mathrm{~dB}$ & $4138 \mathrm{~Hz}$ \\
\hline Pade 2 & $6.66 \mathrm{~dB}$ & $4918 \mathrm{~Hz}$ \\
\hline Pade 3 & $7.24 \mathrm{~dB}$ & $4643 \mathrm{~Hz}$ \\
\hline
\end{tabular}

TABLE II

SENSITIVITY PEAK COMPARISONS

It is also obvious that the proposed controller has more attenuation for disturbances below $6 \mathrm{kHz}$ due to lower sensitivity magnitude.

\section{HDD TMR ANALYSIS}

In this section, we would like to investigate the tracking TMR and tracking TPI capability of the designed servo loop, by applying the disturbances measured from the target HDD. In HDD industries, the major RRO (Repeatable Runout) components of PES (Position Error Signal) are usually treated separately. Therefore the TMR analysis is based on the NRRO components of PES data. Due to the industryspecific modulation/demodulation methods, it is usually very 


$$
C_{\text {opt }}(s)=\frac{10.57 s^{2}+2.01 \times 10^{4} s+2.96 \times 10^{10}}{s^{2}+2.46 \times 10^{5} s+1.55 \times 10^{10}}\left(\frac{1}{1+\frac{4.4 \times 10^{5}}{s+3.98 \times 10^{3}}+H_{F I R}(s)}\right)
$$

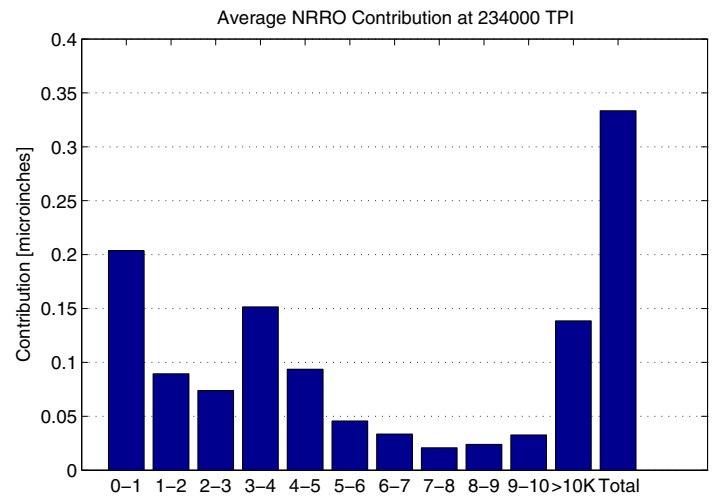

Fig. 6. Frequency components of NRRO: the proposed method.

difficult to get industry PES data for academic research. Most of the existing research results are based on PES data from spin stand environments, which could not fully reflect the actual TMR for servo tracking. In the present paper, we use actual PES data collected from an industry partner.

Figure 6 shows the NRRO components (using $1 k \mathrm{HZ}$ frequency bins) with the $\mathcal{H}^{\infty}$ controller proposed in present paper and similar NRRO spectrum plots are also provided for the case of $1^{\text {st }}$ order, $2^{\text {nd }}$ order, and $3^{\text {rd }}$ orderPadé approximation methods, respectively (as depcited in Figures 7, 8, and 9). It is very clear that the NRRO disc modes from $2 \mathrm{kHz}$ to $5 \mathrm{kHz}$ are better attenuated with the proposed controller, which agrees well with the loop shape comparisons in Figure 5.

A more detailed comparison is given in Table III, where TMR statistics of Mean NRRO value and Mean $+3 \sigma$ NRRO are provided, with the corresponding tracking TPI capabilities. It is shown that $2^{\text {nd }}$ order and $3^{\text {rd }}$ order Padé approximation methods outperform $1^{\text {st }}$ order one by more than $6 \%$ TPI improvement. But higher order Padé approximations will not help further. Note that major issues with higher order Padé approximations are 1) higher order controllers resulted from the models, and 2) undesired zeros/poles introduced by Padé approximations. As a matter of fact, a common practice in industry HDD servo control is still $1^{\text {st }}$ order Padé approximation based method. The data in Table III clearly demonstrates the improvement using the $\mathcal{H}^{\infty}$ control method for infinite dimensional systems, developed in present paper, where more than $10 \%$ TPI improvement can be achieved.

\section{CONCLUDING REMARKS}

In this paper, we have developed a robust control algorithm for HDD servo loop shaping, where the time delay of the model was treated as an infinite dimensional block directly, as opposite to the Padé approximation methods of existing results. The explicit form of the $\mathcal{H}^{\infty}$ controller was

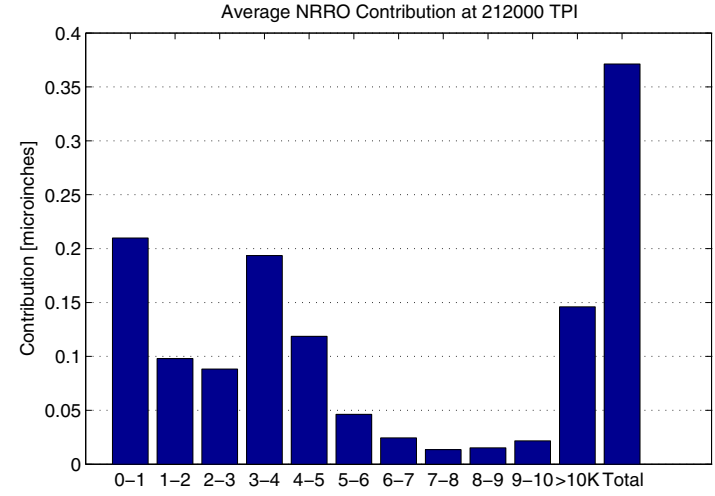

Fig. 7. Frequency components of NRRO: $1^{\text {st }}$ order Padé approximation.

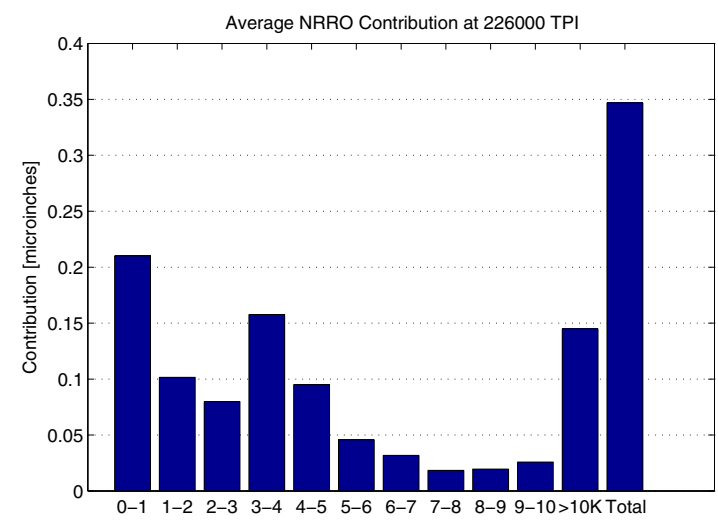

Fig. 8. Frequency components of NRRO: $2^{\text {nd }}$ order Padé approximation.

provided and a internal feedback structure of the controller was also given, which consists of a low order IIR and an FIR. TMR analysis showed significant improvement using the proposed method, with more than $10 \%$ tracking TPI capability achieved.

\section{REFERENCES}

[1] Abramovitch, D., and Franklin, G., 2002. A brief history of disk drive control. IEEE Control Systems Magazine, 22(3), pp.28-42.

[2] Boettcher, U., De Callafon, R. A., and Talke, F. E., 2010. "An internal model-based tracking control for a class of uncertain linear time-varying systems". In Journal of Advanced Mechanical Design, Systems, and Manufacturing, 4(1), pp. 107-118.

[3] Chen, B., Lee, T., and Venkataramanan, V., 2006. Hard Disk Drive Servo Systems. Advances in Industrial Control Series, Springer, New York, NY.

[4] Devasia, S., Eleftheriou, E., and Moheimani, R., 2007. A survey of control issues in nano-positioning. IEEE Transactions on Control Systems Technology, 15(5), pp. 802-823.

[5] Dong, F., and Tomizuka, M., 2009. "An iterative learning control for self-servo writing in hard disk drives using $\mathcal{L}_{1}$ optimal control". In Proceedings of American Control Conference, St. Louis, Missouri, pp 240-245. 


\begin{tabular}{|c|c|c|c|c|}
\hline Methods & NRRO: Mean ( inch) & NRRO: Mean+3 $\sigma(\mu i n c h)$ & TPI & TPI Improvement \\
\hline $1^{\text {st }}$ order Padé approximation method & 0.37 & 0.54 & $212 K$ & N/A \\
\hline $2^{\text {nd }}$ order Padé approximation method & 0.34 & 0.51 & $226 K$ & $6.60 \%$ \\
\hline $3^{r d}$ order Padé approximation method & 0.34 & 0.51 & $225 K$ & $6.13 \%$ \\
\hline Method of the present paper & 0.33 & 0.49 & $234 K$ & $10.38 \%$ \\
\hline
\end{tabular}

TABLE III

COMPARISONS OF TMR AND TPI CAPABILITIES.

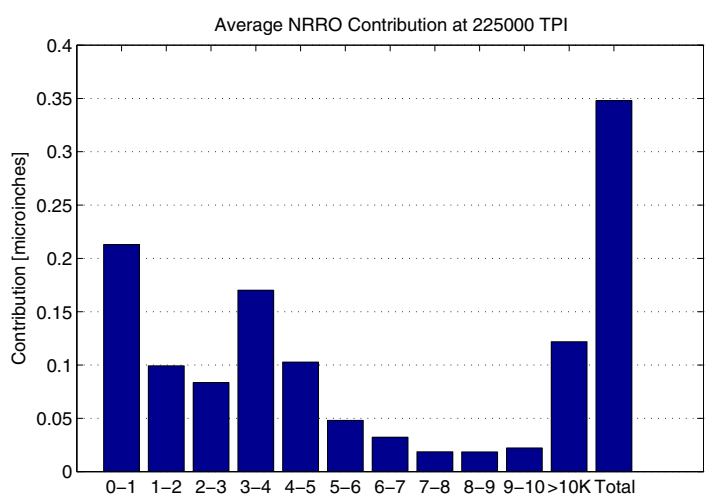

Fig. 9. Frequency components of NRRO: $3^{\text {rd }}$ order Padé approximation. trollers for infinite dimensional SISO plangts". In IEEE Transactions on Automatic Control, 40(4), pp. 751-755.

[20] Wu, S., and Tomizuka, M., 2008. "An iterative learning control design for self-servo writing in hard disk drives". In Proceedings of IFAC World Congress, Seoul, Korea, pp 839-844.

[21] Yan, P., Mckenzie, L., Hanson, R., Lyle, T., Pollock, B., and Mathur, P., 2008. "Servo systems design for low sample rate disk drive systems". In Proceedings of the 18th Annual ASME Conference on Information Storage and Processing Systems, Santa Clara, CA, pp. SCT-B3.

[22] Young, P. M., Morris, J. C., and Ho, H. T., 2006. " $\mu$-synthesis control with PI augmentation". In Proceedings of the American Control Conference, Minneapolis, MN, pp 1297-1302.

[23] Zhong, Q.-C., 2006. Robust Control of Time-delay Systems. SpringerVerlag, London.

[24] Zhou, K., Glover, K., and Doyle, J. C., 1995. Robust and Optimal Control. Prentice-Hall, Englewood Cliffs, NJ.

[25] Zou, Q., Leang, K. K., Sadoun, E., and Reed, M. J, 2004. Control issues in high-speed AFM for biological applications: collagen imaging example. Asian Journal of Control, 6(2), pp.164-178.

[6] Foias, C., Özbay, H., and Tannenbaum, A., 1996. Robust Control of Infinite Dimensional Systems: Frequency Domain Methods. Lecture Notes in Control and Information Sciences, vol. 209, Springer, Berlin.

[7] Gumussoy, S., 2012. "Coprime-inner/outer factorization of SISO timedelay systems and FIR structure of their optimal $\mathcal{H}_{\infty}$ controllers". In International Journal of Robust and Nonlinear Control, 22(3), pp. $981-998$.

[8] Huang, X., and Horowitz, R., 2005. "Robust controller design of a dual-stage disk drive servo system with an instrumented suspension". In IEEE Transactions on Magnetics, 41(8), pp. 2406-2413.

[9] Huang, X., Lee, J.-I., Ramakrishnan, N., Bedillion, M., and Chu, P., 2010. Nano-positioning of an electromagnetic scanner with a MEMS capacitive sensor. Mechatronics, 20(1), pp. 27-34.

[10] Kim, K.-S., Lee, S.-H., and Chung, C.-C., 2011. "A survey of control issues in optical data storage systems". In Proceedings of IFAC World Congress, Milano, Italy, pp 854-868.

[11] Mamun, A. A., Guo, G., and Bi, C., 2007. Hard Disk Drive: Mechatronics and Control. Automation and control engineering, CRC Press, Boca Raton, FL.

[12] Meinsma, G., Mirkin, L., and Zhong, Q.-C., 2002. "Control of systems with I/O delay via reduction to a one-block problem". In IEEE Transactions on Automatic Control, 47(11), pp. 1890-1895.

[13] Messner, W., and Ehrlich, R., 2001. "A tutorial on controls for disk drives". In Proceedings of American Control Conference, Arlington, Virginia, pp 408-420.

[14] Nie, J., and Horowitz, R., 2010. "Control design of concentric selfservo track writing systems for hard disk drives". In Proceedings of American Control Conference, Baltimore, MD, pp 2631-2640.

[15] Ohno, K., and Horowitz, R., 2005. A variable structure multirate state estimate for seeking control of HDDs. IEEE Transactions on Control Systems Technology, 13A(2), pp.233-245.

[16] Pao, L., Franklin, G. F., 1993. "Proximate time-optimal control of third-order servomechanisms". In IEEE Transactions on Automatic Control, 38(4), pp. 560-580.

[17] Pao, L., Franklin, G. F., 1994. "The robustness of a proximate timeoptional controller". In IEEE Transactions on Automatic Control, 39(9), pp. 1963-1966.

[18] Sebastian, A., and Salapaka, S. M., 2005 "Design methodologies for robust nano-positioning". In IEEE Transactions on Control Systems Technology, 13(6), pp. 868-876.

[19] Toker, O., and Özbay, H., 1995. " $\mathcal{H}^{\infty}$ optimal and suboptimal con- 